BASIC RESEARCH

\title{
Non-invasive evaluation of atherosclerosis with contrast enhanced 16 slice spiral computed tomography: results of ex vivo investigations
}

\author{
S Schroeder, A Kuettner, T Wojak, J Janzen, M Heuschmid, T Athanasiou, T Beck, C Burgstahler, \\ C Herdeg, C D Claussen, A F Kopp
}

Heart 2004;90:1471-1475. doi: 10.1136/hrt.2004.037861

See end of article for authors' affiliations

Correspondence to: Priv-Doz Dr Stephen Schroeder, Medical Clinic, Department of Cardiology, Ottried-Mueller-Strasse 10, 72076 Tuebingen, Germany;

stephen.schroeder@ med.uni-tuebingen.de

Accepted 11 June 2004

\begin{abstract}
Objective: To evaluate the diagnostic accuracy of 16 slice computed tomography (CT) in determining plaque morphology and composition in an experimental setting. The results were compared with histopathological analysis as the reference standard.

Methods: Nine human popliteal arteries derived from amputations because of atherosclerotic disease were investigated with multislice spiral CT (MSCT). Atherosclerotic lesions were morphologically classified (completely or partially occlusive, concentric, eccentric), and tissue densities were determined within these plaques. In addition, vessel dimensions were quantitatively measured.

Results: The results were compared with histological analysis. The concordance index $\kappa$ for morphological classification was 0.88 . Plaque density ( $\mathrm{n}=51$ lesions) was significantly different $(\mathrm{p}<0.0001)$ between lipid rich, fibrotic, and calcified lesions (Stary stage III: $n=2,58(8)$ Hounsfield units (HU); Stary V: $\mathrm{n}=11,50$ (21) HU; Stary VI: $\mathrm{n}=14,96$ (42) HU; Stary VII: $\mathrm{n}=6$ 6, 858 (263) HU; Stary VIII: $\mathrm{n}=18$, 126 (99) HU). The concordance index $\kappa$ for the classification of plaques based on density was 0.51 . Vessel dimensions had a good correlation $(r=0.98)$.

Conclusions: 16 slice CT was found to be a reliable non-invasive imaging technique for assessing atherosclerotic plaque morphology and composition. Although calcified lesions can be differentiated from non-calcified lesions, the diagnostic accuracy in further subclassifying non-calcified plaques as lipid rich and fibrotic is low, even under experimental conditions.
\end{abstract}

\section{METHODS}

Nine human popliteal arteries were scanned by 16 slice CT after amputation of the lower limb because of atherosclerotic disease (vessel length $8-14 \mathrm{~cm}$ ). The study protocol was approved by the local ethics committee.

\section{Experimental prestudy}

An experimental prestudy was performed to evaluate the contrast attenuation of various contrast medium-saline dilutions aimed at detecting the correct dilution to achieve a contrast enhancement comparable with those achieved in clinical studies of about 200-250 Hounsfield units (HU). ${ }^{18}$ Silicon tubes (diameter $6 \mathrm{~mm}$, wall thickness $1 \mathrm{~mm}$, lumen diameter $4 \mathrm{~mm}$, wall density $188 \mathrm{HU}$ ) were used to simulate arteries. ${ }^{19}$ These tubes were filled with various contrast medium-saline concentrations ranging from 1:20-1:110. The tubes were scanned by spiral scan technique with $120 \mathrm{kV}, 500 \mathrm{~mA}$, rotation time 0.42 seconds, collimated slice width $16 \times 0.75 \mathrm{~mm}$, and spiral pitch 2.8 . The raw data were reconstructed with an image increment of $0.5 \mathrm{~mm}$, a slice width of $1 \mathrm{~mm}$, and a medium sharp convolution kernel (B30f). Density was measured five times within the contrast enhanced lumen.

\section{Pathological examination}

The popliteal arteries were isolated, prepared, and transported on the day of amputation to the Department of Radiology to be examined by MSCT. The entire vessel was filled under pressure with a contrast medium-saline mixture to achieve accurate filling of the arteries and to prohibit collapsing. After completion of MSCT, the arteries were immediately fixed under pressure, embedded in paraffin,

Abbreviations: $\mathrm{CT}$, computed tomography; $\mathrm{EBCT}$, electron beam computed tomography; HU, Hounsfield units; MSCT, multislice spiral computed tomography; PROCAM, prospective cardiovascular Munster 
histologically sectioned in $5 \mathrm{~mm}$ slices, and stained with haematoxylin and eosin. An experienced observer who was blinded to the MSCT results quantitatively measured vessel diameter and qualitatively analysed plaque morphology and composition. The results were set as the reference standard. All analyses were preformed with a Leica DMLB (Leica, Wetzlar, Germany) microscope. Plaque morphology was classified as follows: completely occlusive, partially occlusive, concentric, and eccentric. Plaques were defined as partially occlusive if they were not circular (defined as concentric) or sickle shaped (defined as eccentric) but had a luminal narrowing. Plaque composition was classified according to the updated eight stage Stary classification..$^{20}$

\section{Computed tomography}

The popliteal arteries were embedded in a bowl filled with ultrasound gel to avoid direct contact with air. A mechanical 16 slice CT machine (Sensation 16, Siemens Medical Solutions, Forchheim, Germany) was used for all investigations. This technology allows for the application of dedicated spiral algorithms that provide a temporal resolution down to $185 \mathrm{~ms}$ and a spatial resolution of up to 11 line pairs $/ \mathrm{cm}$ $(0.5 \times 0.5 \times 0.75 \mathrm{~mm}) .^{5}$ The proximal end of the popliteal artery was cannulated with a 5 French balloon catheter $(6.0 \times 2.0 \mathrm{~mm})$ to seal the proximal vessel segments and to allow the artery to be completely filled with contrast medium. A concentration of contrast medium to saline of 1:80 (Imeron 400; Altana, Konstanz, Germany) was used to achieve optimal contrast attenuation of the popliteal artery (fig 1). The contrast medium was injected continuously at $1.5 \mathrm{~cm}^{3} / \mathrm{s}$ with an injection pump. The scan protocol was adapted to the protocol used for in vivo scanning of the coronary arteries. ${ }^{3}{ }^{4}$ The scan protocol was as follows: $120 \mathrm{kV}, 500 \mathrm{~mA}$, rotation time 0.42 seconds, collimated slice width $16 \times 0.75 \mathrm{~mm}$, and

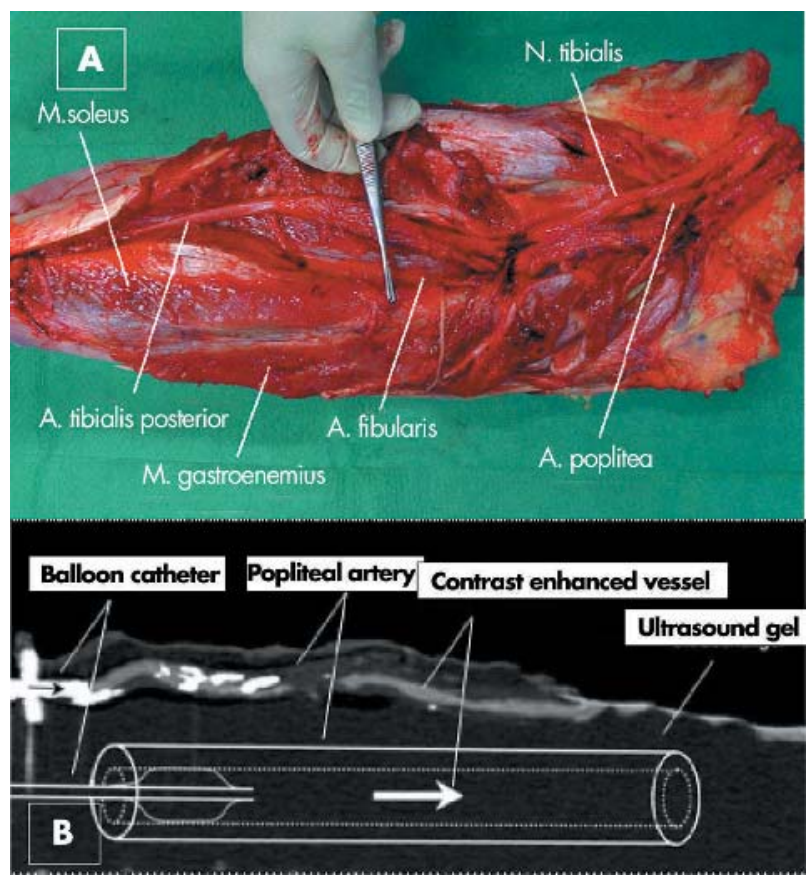

Figure 1 Multislice spiral computed tomography (MSCT) example of a contrast enhanced popliteal artery. (A) Image example of artery preparation for MSCT acquisition. A fibularis, fibular artery; A poplitea, popliteal artery; $A$ tibialis posterior, posterior tibial artery; $M$ gastrocnemius, gastrocnemius muscle; $M$ soleus, soleus muscle; $N$ tibialis, tibial nerve. (B) Contrast enhanced popliteal artery with both calcified and non-calcified plaques. spiral pitch 2.8. In each scan 50-100 axial slices were obtained. The raw data were reconstructed with an image increment of $0.5 \mathrm{~mm}$, a slice width of $1 \mathrm{~mm}$, and a medium sharp convolution kernel (B30f). The matrix size was $512 \times 512$ and the field of view for image reconstruction was set at $100 \times 100 \mathrm{~mm}$. Data were further analysed on an offline workstation (Leonardo, Siemens Medical Solutions).

To facilitate direct comparison between MSCT and histopathological results, the presence of atherosclerotic plaques was evaluated in $5 \mathrm{~mm}$ steps on axial slices (every fifth slice), as well as on maximum intensity projections (650 HU window, $230 \mathrm{HU}$ level). In addition, anatomical orientation points, such as side branches, were used to ascertain exact alignment of MSCT and histological results.

Vessel diameter was quantitatively measured on the selected slices. The morphology of detected plaques was classified as completely occlusive, partially occlusive, concentric, and eccentric. Plaque composition was evaluated by measuring density. Density was measured five times within the predominant tissue of each plaque at five different places with a region of interest ranging from $30-50 \%$ relative to the plaque area. The region of interest was adapted to the plaque size. Each plaque was then characterised by mean and median values. All measurements were taken by one independent observer who was blinded to the histological results.

\section{Statistical analyses}

Continuous variables are presented as their mean (SD) and median. The results of plaque morphology (completely occlusive, partially occlusive, concentric, and eccentric) determined by MSCT were compared with the reference standard histological analysis by contingency analysis. The concordance index $\kappa$ was calculated. Density was measured five times within each plaque area. The comparison of plaque density as determined by MSCT with the histological classification was based on the median of the five density measurements. Box and whiskers plots showing range and quartiles were used to describe the results of the density measurements. Analysis of variance was performed to compare the results of the density measurements of the plaque groups. The nominally scaled histopathological parameters-calcified plaque, fibrotic plaque, lipid rich plaque, and thrombus-and the MSCT measurements were compared by logistic regression analysis. The multivariate model estimated the probability that the density values belonged to one of the nominal values. A contingency analysis was performed to evaluate whether the statistically estimated plaque composition corresponded with the histological reference standard. Probability values of $\mathrm{p}<0.05$ were considered significant. All analyses were performed with JMP 4.0 (SAS Institute, Cary, North Carolina, USA).

\section{RESULTS}

Figure 2 summarises the results of the dilution experiments. The density of the mixture of contrast agent and saline diluted 1:80 was 237 (17) HU. Since attenuation values of 200-250 HU within the contrast enhanced popliteal arteries were desired, this mixture was used for the ex vivo studies.

\section{Morphological classification}

Fifty seven histological sections were evaluated histologically: $16(28 \%)$ were classified by the reference standard as completely occlusive, five (9\%) as partially occlusive, 26 $(46 \%)$ as concentric, and $10(18 \%)$ as eccentric. Because of a technical defect of the MSCT scanner, only 47 of 57 plaque areas could be compared with MSCT: 15 (32\%) were classified as completely occlusive, five (11\%) as partially occlusive, $18(38 \%)$ as concentric, and nine (19\%) as 


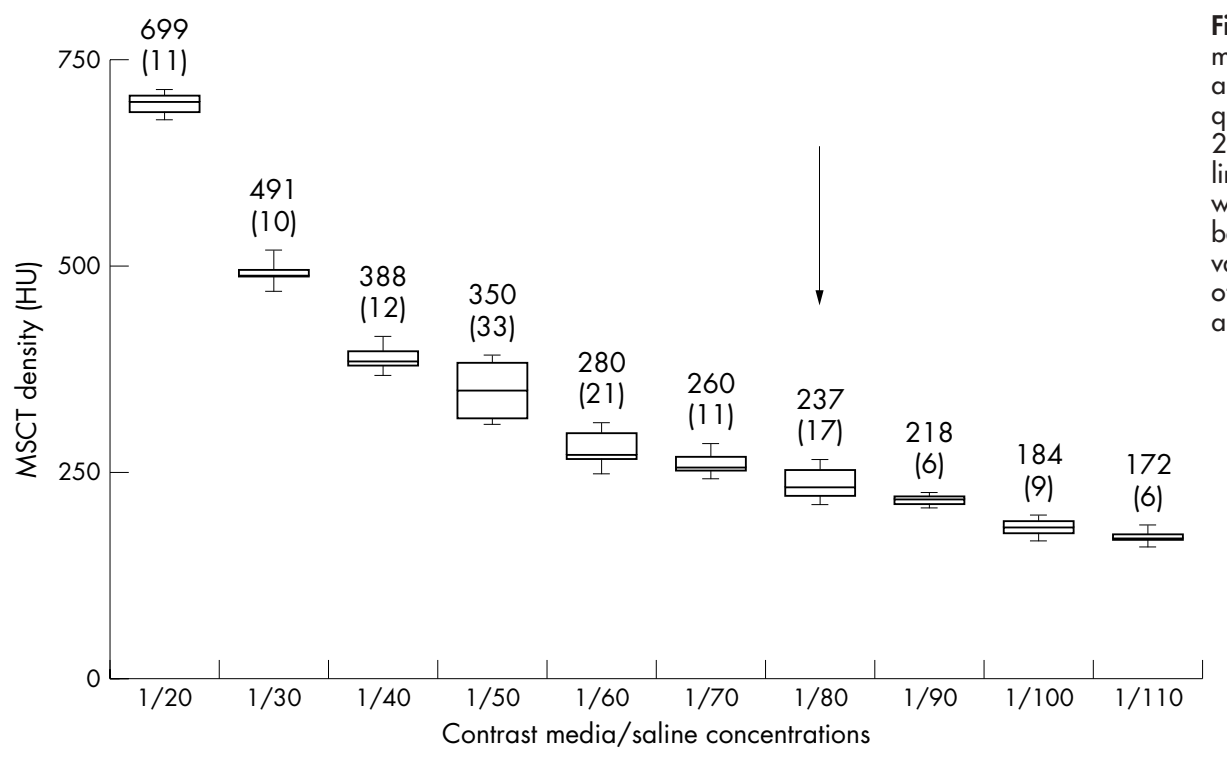

Figure 2 Densities of various contrast medium-saline concentrations in box and whiskers plot showing range and quartiles. The box extends from the 25 th centile to the 75 th centile, with a line at the median (50th centile). The whiskers extend above and below the box to show the highest and lowest values. The arrow indicates the density of a 1:80 dilution. Mean (SD) densities are given. HU, Hounsfield units.

eccentric. The MSCT investigator classified plaque morphology as follows: all complete occlusions were correctly classified, three of five partial occlusions were correctly detected (two were misclassified as eccentric), 17 concentric plaques were correctly classified (one was misclassified as eccentric), eight eccentric were correctly classified (one was misclassified as concentric). The concordance index $\kappa$ for the morphological classification was 0.88 . Sensitivity, specificity, and positive and negative predictive values of MSCT in classifying plaque morphology were as follows: completely occlusive, $100 \%, 100 \%, 100 \%$, and $100 \%$; partially occlusive, $60 \%, 100 \%, 100 \%$, and $94 \%$; concentric, $94 \%, 97 \%, 94 \%$, and $97 \%$; and eccentric, $89 \%, 97 \%, 89 \%$, and $92 \%$, respectively.

Stary V
Histology

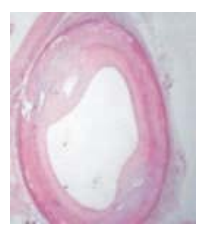

Stary VI
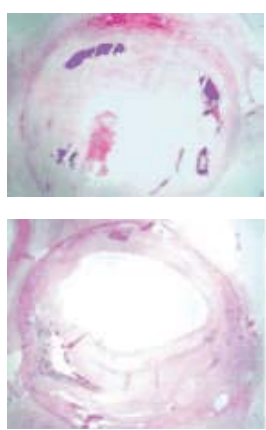

Stary VIII

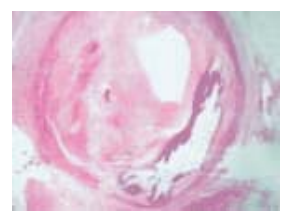

MSCT
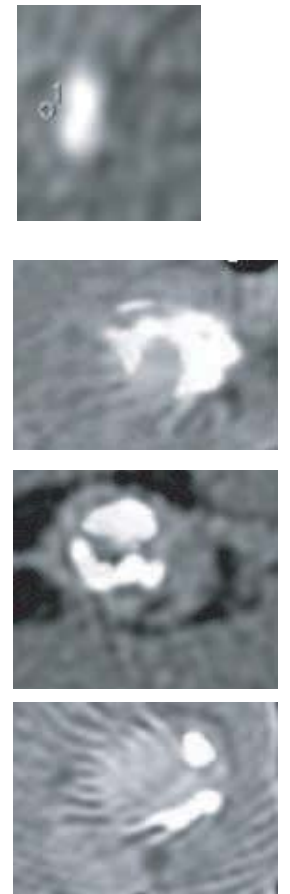

Figure 3 Image examples of Stary stage V-VIII plaques comparing histological with corresponding MSCT slices. Haematoxylin and eosin stain.

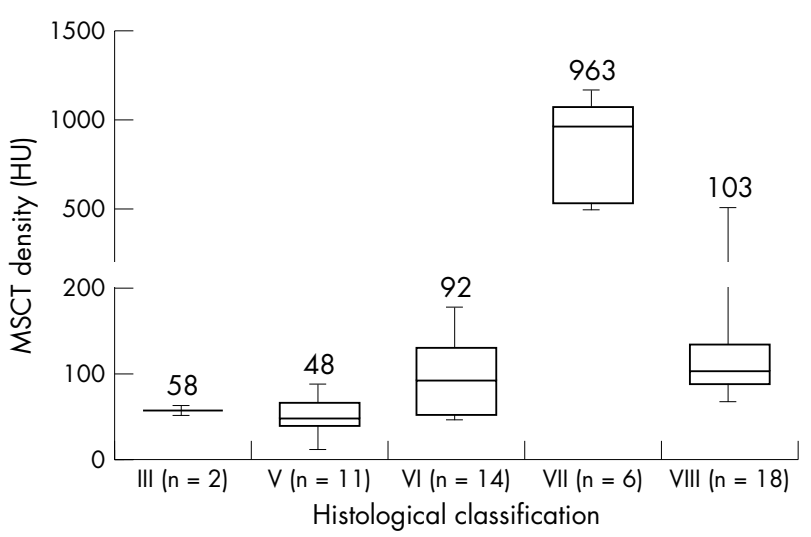

Figure 4 Comparison of histological classification with MSCT density measurements in box and whiskers plot showing range and quartiles. The box extends from the 25 th centile to the 75 th centile, with a line at the median (50th centile). The whiskers extend above and below the box to show the highest and lowest values. Data are medians.

\section{Quantitative measurements}

Quantitative measurements were taken on 84 slices and sites to determine the vessel diameter. The following results were obtained for vessel diameter: 6.44 (1.6) $\mathrm{mm}$ by histological analysis versus 6.92 (1.6) $\mathrm{mm}$ by MSCT (Pearson $r=0.97$, $\mathrm{p}<0.0001)$. Bland-Altman analysis showed a mean difference between the vessel diameters of $0.49(0.36) \mathrm{mm}(95 \%$ confidence interval 0.41 to 0.57 ).

\section{Determination of plaque density}

Plaque composition was histologically evaluated on 51 slices and classified according to $\operatorname{Stary}^{20}$ as follows: two were stage III, 11 were stage V, 14 were stage VI, six were stage VII, and 18 were stage VIII (fig 3). Results of MSCT density measurements within these plaques were as follows: Stary stage III: 58 (8) HU (median 58); stage V: 50 (21) HU (median 48); stage VI: 96 (42) HU (median 92); stage VII: 858 (263) HU (median 963); and stage VIII: 126 (99) HU (median 103). These results was significantly different $(\mathrm{p}<0.0001)$ for lipid rich $(\mathrm{III} / \mathrm{V})$, fibrotic (VIII), and calcified lesions (VII). 


\section{Classification of plaques based on MSCT density values}

As fig 4 shows, an overlap in density values between the different plaque groups was obvious. Thus, a multivariate analysis was performed to evaluate the accuracy of classifying plaque composition based on density measurements. Stary III and V plaques were regarded as lipid rich, VI as thrombi, VII as calcified, and VIII as fibrotic.

All six calcified plaques were classified correctly. Fourteen of 18 fibrotic plaques were classified correctly; one was misclassified as calcified, one as lipid rich, and two as thrombi. Thirteen of 14 lipid rich plaques were classified correctly; one was misclassified as fibrotic. Only one of 14 Stary VI plaques was classified correctly (eight were misclassified as fibrotic and five as lipid rich plaques. The overall concordance index $\kappa$ for the classification of plaques was calculated as 0.51 (without thrombi as nominal parameter, $\kappa=0.56$ ). Sensitivity, specificity, and positive and negative predictive values of MSCT in classifying plaques was as follows: lipid rich, 92\%, 84\%, 67\%, and 97\%; fibrotic, $78 \%, 73 \%, 61 \%$, and $86 \%$; calcified, $100 \%, 98 \%, 86 \%$, and $100 \%$; and thrombi, 7\%, 95\%, 33\%, and $73 \%$, respectively.

\section{DISCUSSION}

The most important findings of the present study are summarised as follows: (1) contrast enhanced MSCT allows for an accurate determination of the morphology of atherosclerotic plaques; (2) tissue density determined within atherosclerotic lesions corresponds well with plaque composition; and (3) our results indicate that plaques with predominantly lipid rich and fibrous tissue have different MSCT attenuation values. However, correct classification is limited because of overlapping test results.

\section{Non-invasive determination of plaque morphology by MSCT}

Various clinical studies focused on the visualisation of intracoronary lesions, indicating a steadily improving diagnostic accuracy of MSCT in detecting clinically relevant stenoses. However, the non-invasive determination of plaque morphology has not been addressed so far. Our results indicate that plaque morphology can be reliably classified by MSCT, at least under experimental conditions with a motionless model. This appears to be of special clinical importance, since both plaque composition and lesion morphology were found to predict plaque stability. Yamagishi et $a l^{21}$ described in an in vivo study with intracoronary ultrasound as the reference standard that eccentric plaques are more likely than concentric plaques to rupture.

\section{Density measurements and plaque composition}

MSCT technology with 16 detector slices allows for noninvasive visualisation of human coronary arteries with diagnostic image quality in vessel segments $>1.5 \mathrm{~mm}$ diameter. ${ }^{4}{ }^{22}$ Immediately after the introduction of MSCTs it became apparent that not only hyperdense but also hypodense areas within contrast enhanced axial slices could be detected..$^{23}$ We recently showed that plaque echogenicity as determined by intracoronary ultrasound corresponds well with plaque density on contrast enhanced four slice CTs. ${ }^{17}$ Since partial volume effects, beam hardening, and iodine attenuation may affect the precision of density measurements in clinical studies, a standardised contrast attenuation of about $200-250 \mathrm{HU}$ is required to assure meaningful and comparable results. ${ }^{19}$

The results of the present study comparing MSCT plaque density measurements with the histopathological reference standard are in keeping with and confirm the initial clinical data. Our results indicate that 16 slice CT allows for noninvasive visualisation of the stages of atherosclerosis. A limitation remains that intracoronary thrombi may be misclassified as non-calcified plaques due to similar attenuation values.

Furthermore, as fig 4 indicates, an overlap of density values between lipid rich and fibrotic plaques is obvious. This had an important impact on the diagnostic accuracy, and only $51 \%$ of all plaques were classified correctly by MSCT criteria. Thus, further subclassification of non-calcified lesions is limited even in this experimental study.

\section{Quantitative measurements with MSCT}

Reliable quantitative measurements within coronary arteries are important for clinical practice-for example, for planning interventions in severe lesions. ${ }^{24-26}$ The presented results are in keeping with initial experimental and clinical reports indicating that quantitative measurement with MSCT is accurate.

\section{MSCT and the vulnerable patient}

Recently Naghavi et $a^{1}{ }^{2}$ discussed the potential value of noninvasive imaging modalities in detecting the vulnerable patient for acute coronary events. The basis of the diagnostic pyramid described was a clinical examination, the patient's history, and the use of established risk scores, such as those used in the Framingham ${ }^{27}$ and PROCAM (prospective cardiovascular Munster) studies. ${ }^{28}$ Since these allow for calculation of relative risk only, implying that only a certain percentage of a patient population obviously is at risk, additional diagnostic efforts are required to characterise the vulnerable patient further. We showed in the present study that MSCT allows for non-invasive detection of the stages of atherosclerosis, including calcified and non-calcified lesions. Thus, this technique may theoretically close the gap between relative risk and actual morphological changes in the arteries.

Leber et $a l^{12}$ recently showed that patients with acute coronary syndromes had a significantly higher number of non-calcified coronary plaques than did patients with stable angina pectoris. We recently showed that high risk patients (PROCAM above the third quintile) without detectable coronary calcifications still have non-calcified plaques with a prevalence of $10 \%{ }^{29}$

Randomised, large scale, prospective studies are needed to determine whether the detection of calcified and noncalcified plaques by MSCT may play a part in the diagnostic algorithm to detect the vulnerable patient.

\section{Limitations of MSCT imaging}

A major limitation of MSCT for its use as a screening test is the high radiation exposure of about $5-10 \mathrm{mSv} .^{30}{ }^{31}$ However, MSCT technology is being further improved with reduced tube current during systole, nearly halving radiation exposure, ${ }^{32}$ as well as the development of scanners with additional detector slices allowing for shorter examination times and presumably stabilised image quality.

Also, the need for iodinated contrast media was reduced to about $80 \mathrm{ml}$ for one scan and is now in the range of conventional coronary angiography. ${ }^{33}$

Other non-invasive imaging modalities such as magnetic resonance imaging and EBCT require no or less radiation. However, no data on a systematic evaluation of non-calcified plaques by EBCT are available, and the value of routine magnetic resonance imaging of plaque is under clinical investigation.

\section{Study limitations}

The current ex vivo study was performed in human popliteal arteries free of motion and breathing artefact to allow for a 
direct comparison of MSCT findings with the histopathological reference standard. The purpose of the study was to evaluate the accuracy of MSCT in differentiating plaque morphology and composition. Since plaque areas on selected slices only and not entire plaques were analysed, we have no information on the sensitivity and specificity of detecting atherosclerotic plaques. In addition, the minimum plaque volume required for MSCT detection is unclear. These issues need to be addressed by future studies, ideally with the use of intracoronary ultrasound in in vivo studies. All popliteal arteries were in advanced stages of atherosclerosis, as opposed to the situation in most patients in whom MSCT would be applied-for example, for screening purposes. The sample was too small to establish reliable thresholds for the differentiation of plaque morphology. As indicated by the mean high density values of fibrotic plaques (126 (99) HU) determining the median (103 HU) may also be useful. This has to be evaluated by further studies with larger sample sizes.

However, since our goal was to evaluate the accuracy of differentiating plaque morphology non-invasively by determining density within plaques by MSCT, our principle conclusions are not affected.

\section{Conclusions}

Sixteen slice CT was found to be a reliable non-invasive imaging technique for assessing the stages of atherosclerosis. Although calcified lesions can be reliably differentiated from non-calcified lesions, the diagnostic accuracy in further subclassifying non-calcified plaques as lipid rich and fibrotic is low, even under experimental conditions.

The role of MSCT in the diagnostic algorithm of vulnerable patients needs to be addressed by further clinical studies.

\section{Authors' affiliations}

S Schroeder*, T Wojak, T Athanasiou, T Beck, C Burgstahler, C Herdeg, Division of Cardiology, Eberhard-Karls-University Tuebingen, Tuebingen, Germany

A Kuettner*, M Heuschmid, C D Claussen, A F Kopp, Division of

Diagnostic Radiology, Eberhard-Karls-University Tuebingen

J Janzen, Department of Pathology, Eberhard-Karls-University Tuebingen

*Both authors contributed equally

This study was supported by a grant from the AKF-Program (70-0-0), University of Tuebingen, and by a grant from Pfizer $\mathrm{GmbH}$, Karlsruhe (ATV-D-01-014G), Germany

\section{REFERENCES}

1 Naghavi $M$, Libby $P$, Falk E, et al. From vulnerable plaque to vulnerable patient: a call for new definitions and risk assessment strategies. Part II. Circulation 2003;108:1772-8.

2 Naghavi $M$, Libby $P$, Falk $E$, et al. From vulnerable plaque to vulnerable patient: a call for new definitions and risk assessment strategies. Part I. Circulation 2003;108:1664-72.

3 Nieman K, Cademartiri F, Lemos PA, et al. Reliable noninvasive coronary angiography with fast submillimeter multislice spiral computed tomography. Circulation 2002;106:2051-4.

4 Ropers D, Baum U, Pohle K, et al. Detection of coronary artery stenoses with thin-slice multi-detector row spiral computed tomography and multiplanar reconstruction. Circulation 2003;107:664-6.

5 Heuschmid M, Kuttner A, Flohr T, et al. [Visualisation of coronary arteries in $\mathrm{CT}$ as assessed by a new 16 slice technology and reduced gantry rotation time: first experiences]. Rofo 2002;174:721-4. (In German.)

6 Achenbach S, Daniel WG. Noninvasive coronary angiography: an acceptable alternative? N Engl J Med, 2001;345:1909-10.

7 Kim WY, Danias PG, Stuber M, et al. Coronary magnetic resonance angiography for the detection of coronary stenoses. N Engl J Med 2001;345:1863-9.
8 Achenbach S, Moshage W, Ropers D, et al. Value of electron-beam computed tomography for the noninvasive detection of high-grade coronary-artery stenoses and occlusions. N Engl J Med 1998;339:1964-71.

9 Rumberger JA. Tomographic plaque imaging with CT: technical considerations and capabilities. Prog Cardiovasc Dis 2003;46:123-34.

10 Becker CR, Schoepf UJ, Reiser MF. Coronary artery calcium scoring: medicine and politics. Eur Radiol 2003;13:445-7.

11 Caussin C, Ohanessian A, Lancelin B, et al. Coronary plaque burden detected by multislice computed tomography after acute myocardial infarction with near-normal coronary arteries by angiography. Am J Cardiol 2003;92:849-52.

12 Leber AW, Knez A, White CW, et al. Composition of coronary atherosclerotic plaques in patients with acute myocardial infarction and stable angina pectoris determined by contrast-enhanced multislice computed tomography Am J Cardiol 2003;91:714-8

13 Nikolaou K, Sagmeister S, Knez A, et al. Multidetector-row computed tomography of the coronary arteries: predictive value and quantitative assessment of non-calcified vessel-wall changes. Eur Radiol 2003; 13:2505-12.

14 de Feyter PJ, Nieman K. New coronary imaging techniques: what to expect? Heart 2002:87:195-7.

15 Gerber TC, Kuzo RS, Lane GE, et al. Image quality in a standardized algorithm for minimally invasive coronary angiography with multislice spiral computed tomography. J Comput Assist Tomogr 2003;27:62-9.

16 Becker CR, Nikolaou K, Muders M, et al. Ex vivo coronary atherosclerotic plaque characterization with multi-detector-row CT. Eur Radiol 2003;13:2094-8

17 Schroeder S, Kopp AF, Baumbach A, et al. Noninvasive detection and evaluation of atherosclerotic coronary plaques with multislice computed tomography. J Am Coll Cardiol 2001;37:1430-5.

18 Kopp AF, Schroeder S, Kuettner A, et al. Non-invasive coronary angiography with high resolution multidetector-row computed tomography: results in 102 patients. Eur Heart J 2002;23:1714-25.

19 Schroeder S, Flohr T, Kopp AF, et al. Accuracy of density measurements within plaques located in artificial coronary arteries by $X$-ray multislice $\mathrm{CT}$ : results of a phantom study. J Comput Assist Tomogr 2001;25:900-6.

20 Stary HC. Natural history and histological classification of atherosclerotic lesions: an update. Arterioscler Thromb Vasc Biol 2000;20:1177-8.

21 Yamagishi M, Terashima M, Awano K, et al. Morphology of vulnerable coronary plaque: insights from follow-up of patients examined by intravascular ultrasound before an acute coronary syndrome. J Am Coll Cardiol 2000;35:106-11

22 Nieman K, van der Lugt A, Pattynama PM, et al. Noninvasive visualization of atherosclerotic plaque with electron beam and multislice spiral computed tomography. J Interv Cardiol 2003;16:123-8.

23 Schroeder S, Kopp AF, Baumbach A, et al. Non-invasive characterisation of coronary lesion morphology by multi-slice computed tomography: a promising new technology for risk stratification of patients with coronary artery disease. Heart 2001;85:576-8.

24 Ferencik M, Moselewski F, Ropers D, et al. Quantitative parameters of image quality in multidetector spiral computed tomographic coronary imaging with submillimeter collimation. Am J Cardiol 2003;92:1257-62.

25 Achenbach S, Ulzheimer S, Baum U, et al. Noninvasive coronary angiography by retrospectively ECG-gated multislice spiral CT. Circulation 2000;102:2823-8.

26 Schroeder S, Kopp AF, Baumbach A, et al. Non-invasive detection of coronary lesions by multi-slice computed tomography: results of the new-age pilot trial. Catheter Cardiovasc Interv $2001 ; 53: 352-8$.

27 Broedl UC, Geiss HC, Parhofer KG. Comparison of current guidelines for primary prevention of coronary heart disease: risk assessment and lipidlowering therapy. J Gen Intern Med 2003;18:190-5.

28 Assmann G, Cullen P, Schulte H. Simple scoring scheme for calculating the risk of acute coronary events based on the 10-year follow-up of the prospective cardiovascular Munster (PROCAM) study. Circulation 2002;105:310-5

29 Schroeder S, Kuettner A, Kopp AF, et al. Noninvasive evaluation of the prevalence of noncalcified atherosclerotic plaques by multi-slice detector computed tomography: results of a pilot study. Int J Cardiol 2003:92:151-5.

30 Achenbach S, Ropers D, Regenfus $M$, et al. Noninvasive coronary angiography by magnetic resonance imaging, electron-beam computed tomography, and multislice computed tomography. Am J Cardiol 2001;88:70E-3E.

31 Achenbach S, Giesler T, Ropers D, et al. Detection of coronary artery stenoses by contrast-enhanced, retrospectively electrocardiographically-gated, multislice spiral computed tomography. Circulation 2001:103:2535-8.

32 Jakobs TF, Becker CR, Ohnesorge B, et al. Multislice helical CT of the heart with retrospective ECG gating: reduction of radiation exposure by ECGcontrolled tube current modulation. Eur Radiol 2002;12:1081-6.

33 Becker C, Schatzl M, Feist $\mathrm{H}$, et al. [Assessment of the effective dose for routine protocols in conventional $\mathrm{CT}$, electron beam $\mathrm{CT}$, and coronary angiography]. Rofo 1999;170:99-104. (In German.) 